\title{
Analysis on the Causes of Cracking at the Last Stage Blade of the Low-pressure Rotor in thermal power plant
}

\author{
Liming Xie ${ }^{1}$ Feng Tian $^{1}$ Jun Liu $^{1}$ Hao Chen ${ }^{1}$ \\ ${ }^{1}$ Inner Mongolia Power Research Institute, Hohhot, Inner Mongolia, 010020, China
}

\begin{abstract}
Transverse cracking occurred in the blade body of the last stage of the low pressure rotor of a 200MW unit in a thermal power plant. The causes of blade cracking were analyzed by means of macro morphology, SEM, microstructure, mechanical properties, chemical composition and energy spectrum analysis. The results show that the corrosive $\mathrm{Cl}^{-}$accumulates on leaves, and then combined action of tensile stress is formed with the static load and dynamic load during the blade operation, and the stress corrosion microcracks had been conceived in the $\mathrm{Cl}^{-}$corrosion region. Under the long period cyclic excitation stress of the blade during the rotor rotation at high speed, the crack source extends in fatigue mode, finally resulting in cracking.
\end{abstract}

\section{Introduction}

The blade is an important part of the power turbine to complete the energy transformation. The turbine blades are working with poor conditions, high-speed rotate in high-temperature and high-pressure media environment for long period, with considerable stress. The turbines of the power station have multi-stage blades and multiple blades for each stage, as long as there is a problem with one of the blades, it is possible to have an accident, which will result in the outage of the unit, and bring about significant economic losses ${ }^{[1][8]}$.

There is a $200 \mathrm{MW}$ unit in a thermal power plant found that several transverse cracking occurred in the blade body of the turbine's low-pressure rotor at the first and last stages. This turbine was produced by the Harbin turbine company limited, whose model is C145/N200-12.7/535/535, ultra-high pressure, one-time intermediate reheat, three-cylinder two-exhaust, single-draw condensed steam. The main steam temperature of the machine is $535^{\circ} \mathrm{C}$, the main steam pressure is $12.75 \mathrm{MPa}$, the reheat steam temperature is $535^{\circ} \mathrm{C}$, and the reheat steam pressure is $2.18 \mathrm{MPa}$. The material of blade is $2 \mathrm{Cr} 13$. The cracking of the last stage blade brings great threat to the safe and stable operation of the unit.

This paper analyzes the causes of the cracking of the turbine blades and puts forward some suggestions to prevent the recurrence of the same type of events and improve the safety and reliability of the unit operation.

\section{Analysis and test for cracking at the last stage blade of turbine}

\subsection{Macro-observation and analysis}

It can be seen from the results of penetration test at the production site that cracking occurs in the last stage blades, the cracking position is located near the tension bar between the blade tension bar and the blade root, and the crack is cracked perpendicular to the blade length direction, as shown in figure 1.

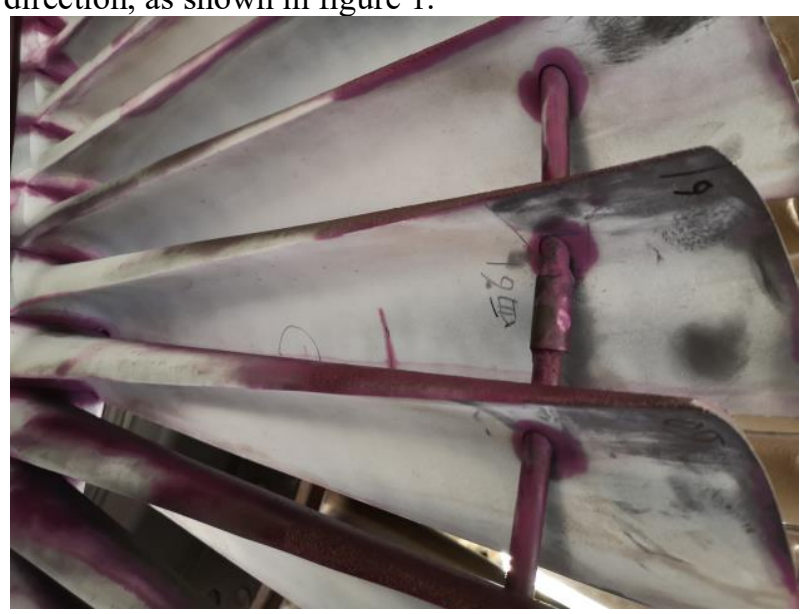

Figure 1 Photos of blade penetration test on site

A large number of corrosion pits were found on the surface of the cracked blade. Select a blade with severe cracking and open the cracking part for macroscopic appearance inspection. The fracture surface of the blade is flush without obvious plastic deformation and defects such as mechanical damage. The fracture surface is a 
typical fatigue fracture, and the initial fracture area and the crack propagation area are clearly distinguishable, the cracking originates from the edge arc of the outlet side of the blade and extends to the inlet side. The direction of cracking is perpendicular to the direction of blade length. The area of crack initiation area is small, and most of the fracture area is expansion area, with typical "beach like" fatigue strip morphology, as shown in Figure $2^{[2]}$.
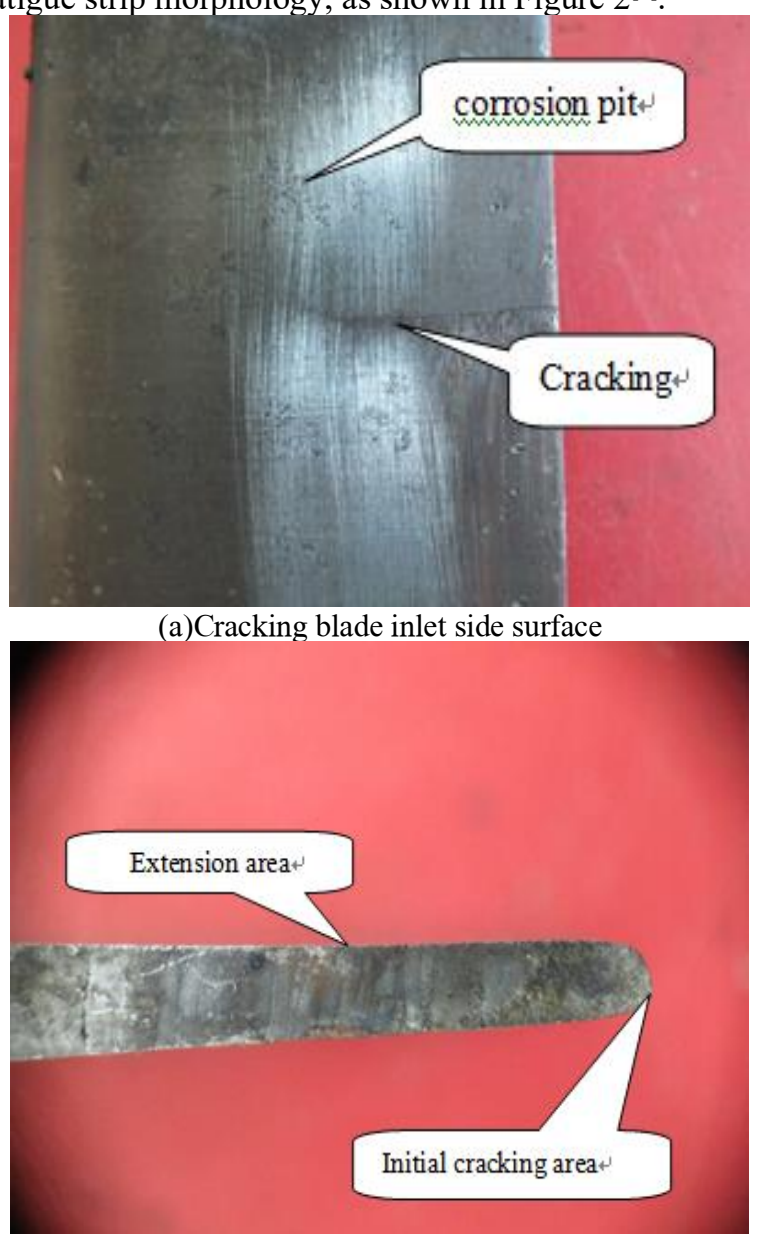

(b)Fracture of the cracked blade

Figure 2 Surface and fracture macro-observation of the cracked blade

\subsection{SEM analysis of fracture surface}

The fracture surface was observed by scanning electron microscope (SEM). It can be seen that the initial fracture area of fracture surface presents a typical intergranular crack morphology of "ice sugar shape". The grains are relatively small, and there are many intergranular cracks associated with it. Corrosion pits at the edge of the fracture in near fracture area, the depth of the corrosion pit is about $0.2 \mathrm{~mm}$, obvious "mud pit" morphology can be observed inside the corrosion pit, it's the typical stress corrosion characteristics. The fatigue striation can be observed in the expansion region, which has a typical characteristic morphology of martensitic steel fatigue expansion, as shown in Figure $3^{[3][4]}$.

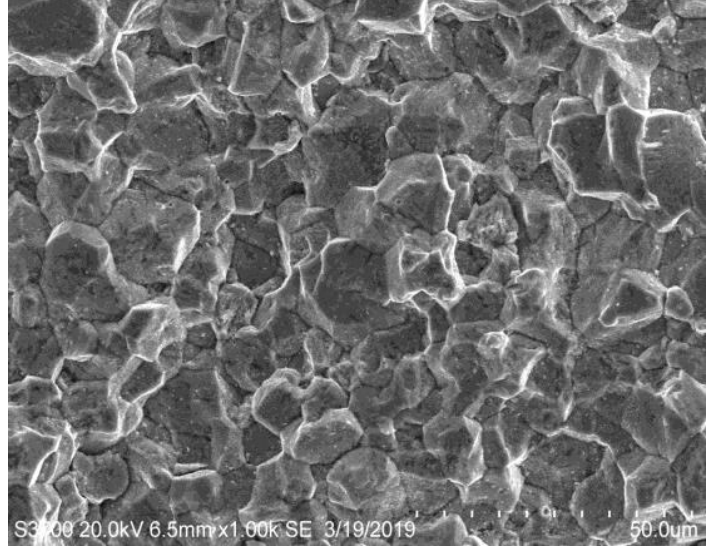

(a)initial fracture area

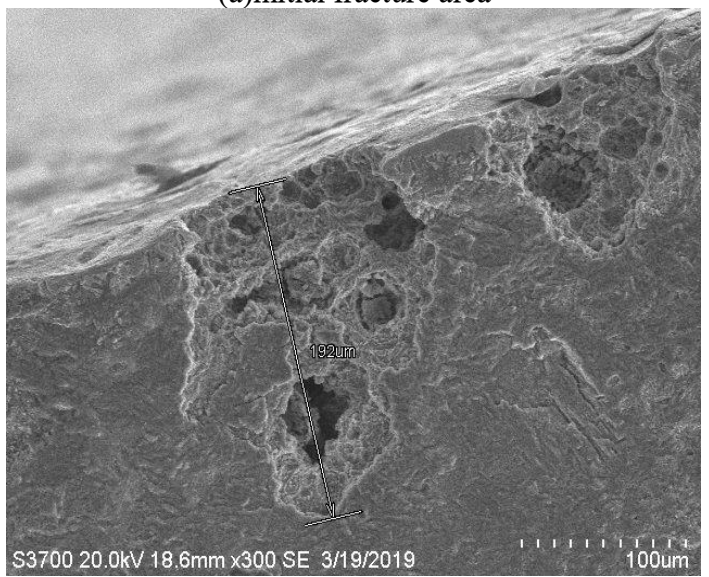

(b)Corrosion pits at fracture edges

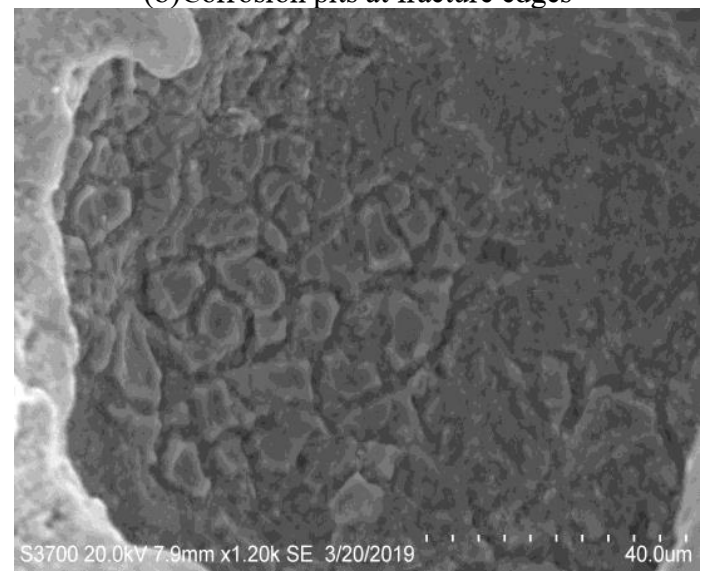

(c)"mud pit" morphology in the corrosion pits

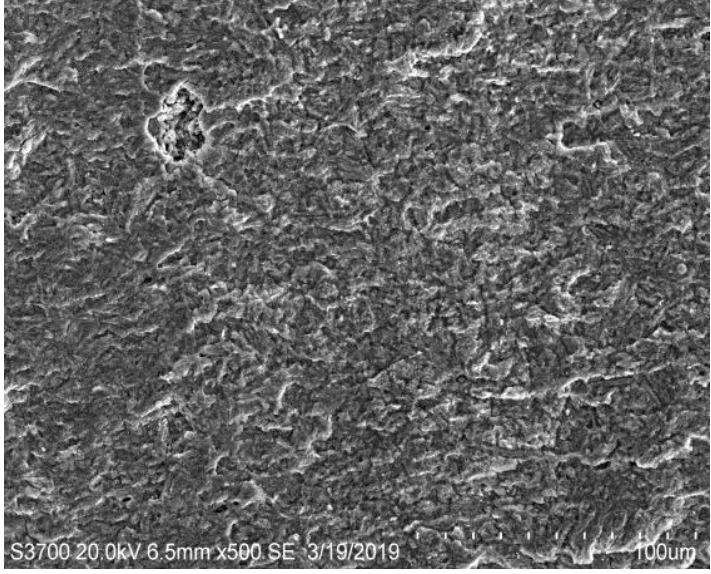

(d)Extension area

Figure 3 The SEM morphology of blade fracture 


\subsection{Energy spectrum detection in fracture}

The fracture of the last stage blade of cracked low-pressure rotor is analyzed in energy spectrum detection. It can be seen from the test results that there are corrosive $\mathrm{Cl}^{-}$ions what is sensitive to martensitic stainless steel in the corrosion pit, and the mass percentage of $\mathrm{Cl}^{-}$ions is $0.38 \%$. The last blade is in the wet steam region, Because of the poor quality of vapor, the vapor carries harmful $\mathrm{Cl}^{-}$, causing pitting corrosion on the weak parts of the blade surface. When the corrosion develops to a certain extent, a fatigue crack source is formed, which eventually leads to blade cracking $^{[5]}[6]$.

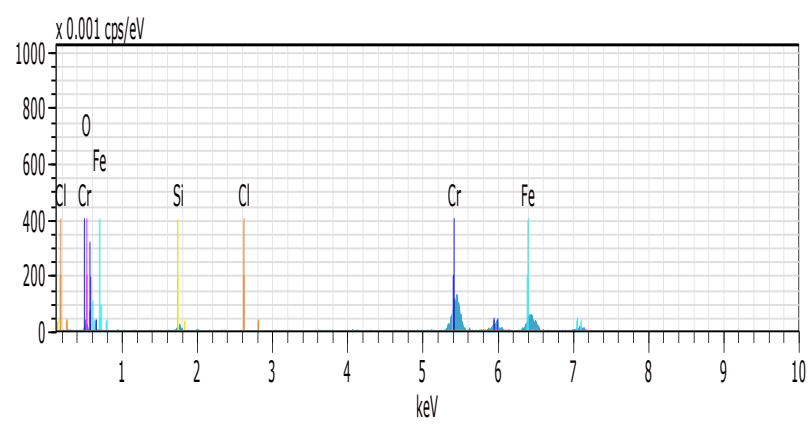

Figure 4 The EDS map of blade fracture

\subsection{Microstructure detection and analysis}

Microstructure detection is used in surface cracking at the last stage blade of low-pressure rotor. It can be seen that the microstructure of the blade is uniformly distributed and fine tempered martensite. There is no abnormal structure such as coarse quenched martensite and no serious inclusion defect in the structure. However, there are a lot of corrosion pits in the structure, and there are crack defects derived from corrosion pits, as shown in Figure $5^{[7]}$.

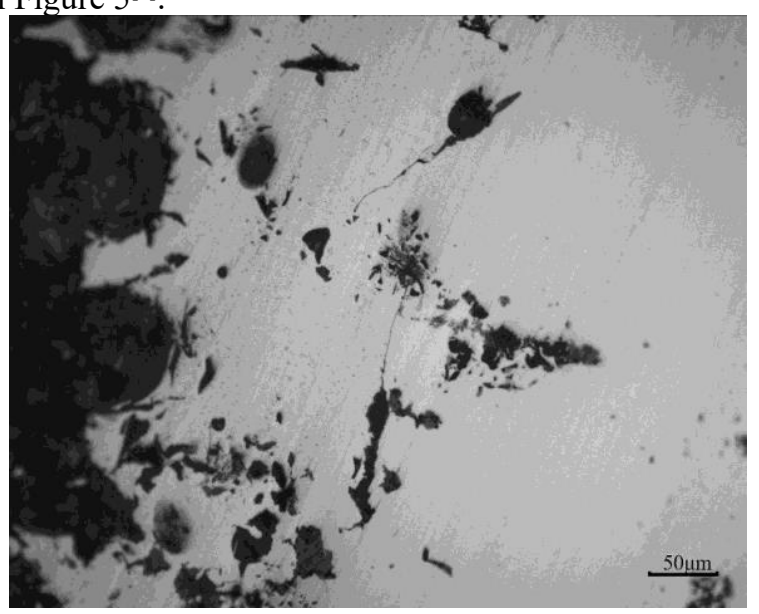

Figure 5 The microstructure of cracked blade

\subsection{Chemical composition Analysis}

The chemical composition of cracked low-pressure last stage blade was tested, the results are shown in table 1 . It can be seen from the results, the contents of each element in the chemical composition of the blade met the requirements for $2 \mathrm{Cr} 13$ (GB/T 8732-2014 Steel for turbine blades) ${ }^{[9]}$.

Table 1 The results of chemical composition test of blade

\begin{tabular}{ccccccccc}
\hline items & $\mathrm{C}$ & $\mathrm{Si}$ & $\mathrm{Mn}$ & $\mathrm{Cr}$ & $\mathrm{Ni}$ & $\mathrm{Cu}$ & $\mathrm{S}$ & $\mathrm{P}$ \\
\hline $\begin{array}{c}\text { measured } \\
\text { value }\end{array}$ & 0.19 & 0.29 & 0.26 & 13.00 & 0.17 & 0.18 & 0.005 & 0.028 \\
$\begin{array}{c}\text { standard } \\
\text { value }\end{array}$ & $\begin{array}{c}0.16 \sim \\
0.24\end{array}$ & $\leq 0.60$ & $\leq 0.60$ & $\begin{array}{c}12.00 \sim \\
14.00\end{array}$ & $\leq 0.60$ & $\leq 0.30$ & $\leq 0.020$ & $\leq 0.030$ \\
\hline
\end{tabular}

\subsection{Mechanical property testing and analysis}

The hardness and impact toughness of the last stage blade of cracked low-pressure rotor were tested, The results are shown in table 2 . It can be seen from the results, the hardness and impact toughness of the last stage blade of cracked low-pressure rotor met the requirements for $2 \mathrm{Cr} 13(\mathrm{~GB} / \mathrm{T} \quad 8732-2014$ steel for turbine blades) ${ }^{[9]}$.

Table 2 The results of mechanical property testing of blade

\begin{tabular}{ccc}
\hline items & hardness $/$ HBW & impact toughness $/ \mathrm{J}$ \\
\hline measured value & 243 & 56 \\
standard value & $212 \sim 262$ & $\geq 27$ \\
\hline
\end{tabular}

\section{Comprehensive analysis}

According to the analysis of fracture morphology, there are a lot of corrosion pits on the surface of the last stage blades of low pressure rotor. Blade cracking originates from the edge of the outlet side of the blade and extends to the inlet side. The cracking direction is perpendicular to the length of the blade. The initial cracking area is small and the surface is rough, most of the fractures are expansion area, where have the typical "beach-like" fatigue strip morphology, the spacing of fatigue strip is 
small, what show that the stress of the blade is smaller, it's provided with the property of high-cycle low-stress fatigue expansion. From the fracture appearance, the fracture initial fracture area shows a typical "ice sugar-like" intergranular cracking morphology. Corrosion pits at the edge of the fracture in the near fracture area, obvious "mud pit" morphology can be observed inside the corrosion pit, and the presence of corrosive $\mathrm{Cl}^{-}$ions what is sensitive to martensitic stainless steel is inside the corrosion pit, it's typical stress corrosion characteristics.

According to the analysis of microstructure, the microstructure of the last stage blade of the low-pressure rotor is equiaxed uniformly distributed, exiguous tempered martensite microstructure. There is no abnormal structure such as coarse quenched martensite and no serious inclusion defect in the structure. However, there are a lot of corrosion pits in the structure, and there are crack defects derived from corrosion pits.

According to the analysis of chemical composition, the content of each element in the chemical composition of the leaf material meets the standard requirements.

According to the analysis of mechanical property testing, the hardness of the last stage blade of cracked low-pressure rotor met the requirements, and the toughness is also enough.

According to the analysis of stress situation, the last stage blade of low-pressure rotor has long blade shape and complex stress in the process of high-speed rotation. In addition to the strong tensile stress static load caused by centrifugal force generated by high-speed rotation, it also has the alternating stress caused by forced vibration caused by uneven steam flow. Under the combined action of strong tensile stress and corrosive $\mathrm{Cl}^{-}$will create stress corrosion cracking in the blade surface, and the forced vibration and even the strong alternating load stress produced by the blade resonance will force the stress corrosion crack to expand in the way of fatigue cracking, which eventually lead to the blade cracking.

\section{Conclusions and recommendations}

According to the test and analysis, the main reason for the cracking of the second last stage blade of the steam turbine is that the steam medium of the unit contains $\mathrm{Cl}^{-}$ which is sensitive to the corrosion of martensitic stainless steel. The corrosive $\mathrm{Cl}^{-}$accumulates on the blade for a long time and forms the tensile stress together with the static load and dynamic load during the operation of the blade. The microcracks of stress corrosion appear in the $\mathrm{Cl}^{-}$corrosion area. Under the long-term cyclic vibration stress produced in the process of high-speed rotor rotation, the stress corrosion crack source propagates in the way of fatigue, which eventually leads to serious cracks in the blade.

In view of the cracks in many places of the last stage blades of the steam turbine of the unit, which pose a great threat to the safe and stable operation of the unit, it is recommended to replace all the cracked blades. First of all, Other blades shall be checked for similar cracking, and problems found shall be handled in time.
Additionally, the quality control of steam water should be strengthened, prevent the corrosive $\mathrm{Cl}^{-}$in steam from gathering and forming punctate corrosion pits in the weak parts on the blade surface. Finally, during the maintenance, it is important to check whether there is any defect of individual nozzle, large deviation of processing and installation, different pitch, bad diaphragm structure and installation, uneven dynamic balance of rotor, to increase the exciting stress of blades, so as to avoid similar cracking accidents again.

\section{Acknowledgments}

I really thanks the help and encouragement from my colleagues in my laboratory, thank you for my family's encouragement, and appreciate Inner Mongolia Electric Power Research Institute for supporting my project fund. (Research on key technologies of turbine blade inspection based on phased array technology, 510241190010)

\section{References}

1. Wu, y.k. (1999) The failure mode and research status of turbine blade. The Technology of Steam Turbine Technology, 37(6):362-365.

2. Chen, c.y. (2002) Fatigue and fracture. Huazhong University of Science and Technology Press, WuHan.

3. Chen, y.y., Lin, z.k. (1981) The corrosion fatigue for the material $2 \mathrm{Cr} 13$ of Steam turbine blade. Materials for Mechanical Engineering, 5(1):36-41.

4. Li, h.h., Lu, z.m., Liu, k. (2017) Analysis of Reasons for Cracking of Steam Turbine Blades in Thermal Power Plant. Physical and chemical testing physical volume, 53(3):197-200.

5. $\mathrm{Xu}$, h. (1991) Mechanical design manual. China Machine Press, Beijin.

6. Zhong, j.y. (1992) Study on fatigue performance of steel for turbine blade. Xi'an Jiaotong University, 32(5)6-12.

7. Yue, m., Li, s.c. (2013) Analysis of Crack Cause of Stainless Steel 2Cr13 Blade, Equipment manufacturing, 13(3): 30-31.

8. National Energy Administration. (2016). DL/T 438-2016 China Electricity Council.

9. General administration of quality supervision, inspection and quarantine of the people's republic of china. (2016). GB/T 8732. China Standardization Administration Committee. 